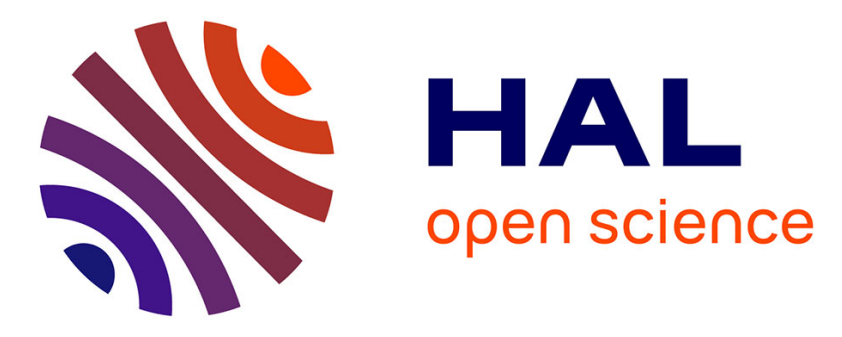

\title{
Digital Control of MEMS Gyroscopes: a Robust Approach
}

\author{
Fabricio Saggin, Cécile Pernin, Anton Korniienko, Gérard Scorletti, \\ Christophe Le Blanc
}

\section{- To cite this version:}

Fabricio Saggin, Cécile Pernin, Anton Korniienko, Gérard Scorletti, Christophe Le Blanc. Digital Control of MEMS Gyroscopes: a Robust Approach. INERTIAL, Mar 2021, Virtual, United States. 10.1109/INERTIAL51137.2021.9430470 . hal-03202412

\section{HAL Id: hal-03202412 \\ https://hal.science/hal-03202412}

Submitted on 19 Apr 2021

HAL is a multi-disciplinary open access archive for the deposit and dissemination of scientific research documents, whether they are published or not. The documents may come from teaching and research institutions in France or abroad, or from public or private research centers.
L'archive ouverte pluridisciplinaire HAL, est destinée au dépôt et à la diffusion de documents scientifiques de niveau recherche, publiés ou non, émanant des établissements d'enseignement et de recherche français ou étrangers, des laboratoires publics ou privés. 


\title{
Digital Control of MEMS Gyroscopes: a Robust Approach
}

\author{
Fabrício Saggin*, Cécile Pernin*, Anton Korniienko*, Gérard Scorletti* and Christophe Le Blanc ${ }^{\dagger}$ \\ ${ }^{*}$ Laboratoire Ampère, Ecole Centrale de Lyon, Université de Lyon, France \\ $\dagger$ Asygn, Grenoble, France
}

\begin{abstract}
This work presents a new approach to design the controllers for MEMS gyroscopes based on the robust $H_{\infty}$ synthesis. A systematic and flexible method for designing digital controllers for the drive and sense modes of a Coriolis vibratory gyroscope is proposed. Furthermore, the sinusoidal signals are directly controlled instead of their amplitude and phase, so that (de)modulation is not required in the control loops. This fact allows us to simplify the electronic design and to provide formal guarantees of stability and performance. First practical results are presented, proving the implementability of our approach.

Index Terms-Direct control, $H_{\infty}$ synthesis, MEMS gyroscope, digital control.
\end{abstract}

\section{INTRODUCTION}

MEMS gyroscopes are microdevices used to measure the angular rate of objects. Their working principle relies on the oscillations of a proof mass along two perpendicular axes $-\vec{x}$ and $\vec{y}-$, defining the so-called drive and sense modes. Oscillations are driven along the $\vec{x}$-axis. If the device is submitted to an angular rate $\Omega_{z}$ (perpendicular to $\vec{x}$ and $\vec{y}$ ), a Coriolis force, proportional to $\Omega_{z}$, appears, provoking oscillations along the $\vec{y}$-axis. Therefore, if the Coriolis force can be estimated, $\Omega_{z}$ can be computed [1]. For an accurate measure of $\Omega_{z}$, control loops are required to $(i)$ regulate the amplitude and phase of the oscillations on the drive mode, and (ii) compensate for the forces acting on the sense mode, producing an estimate of the Coriolis force.

In the literature, several control strategies are proposed to fulfill the above requirements. For the drive mode control, we can mention: the automatic gain control (AGC) combined with a phase-locked loop (PLL), see, e.g., [1], [2]; and the selfoscillating AGC, see, e.g., [3]. For the sense mode closed-loop operation, the most widespread architectures are: the in-phase and in-quadrature compensation loops, see, e.g., [1], [2]; and the electro-mechanical $\Sigma \Delta(\mathrm{EM}-\Sigma \Delta)$, see [4], [5].

In general, these control architectures have the advantage of being of a simple implementation. In addition, except for the EM- $\Sigma \Delta$, they work with low-frequency signals (envelopes or baseband signals), such that the control objectives can be cast as to track or reject constant signals. Hence, PI-like controllers can be employed [1]-[3]. In the particular case of the EM- $\Sigma \Delta$, the controller design, inspired by the classical $\Sigma \Delta$ converters, follows a more complex but still accessible framework [4].

On the other hand, the main drawback of these nonlinear strategies is the lack of performance and stability guarantees.

This work was supported by BPI France.
In general, drive and sense modes, despite the mechanical coupling between them, have control loops independently designed. Hence, there are no guarantees that when the drive and sense loops are closed, the whole system will be stable and achieve the desired performance. Moreover, the presence of nonlinear elements in the loops (such as relays, modulators or mixers, envelope detector) requires, for the controller design, a model linearization [1], [3], [4], which is often valid for a single operating point.

An alternative approach is to consider a classical feedback architecture (from the Control Theory point of view), which we name direct control. In this architecture, the controller is directly connected to the MEMS gyroscope, without nonlinear elements in between. Besides, since the MEMS gyroscope can be modeled as a linear system [1], [6], well-established control design methods can be employed, including multivariable ones. Thus, the couplings between drive and sense modes can be adequately considered, providing guarantees of stability and performance for the overall closed-loop system.

The use of the direct control architecture for MEMS gyroscopes is not new, see, e.g., [6], [7]. However, practical results are rarely reported in the literature, which might indicate issues with the implementation of the proposed solutions.

In this work, we propose a design method for a multivariable digital controller based on the celebrated $H_{\infty}$ synthesis and the direct control architecture. The proposed solution is implemented on the platform AS3125-SDK, operating with an experimental MEMS gyroscope, to demonstrate the implementability and the validity of the proposed approach.

The paper is organized as follows. Section II recalls the operating principles of the MEMS gyroscopes and details the direct control architecture. Section III presents the $H_{\infty}$ synthesis for the digital controller design. Section IV presents the implementation results, while Section V draws the conclusions and perspectives of this work.

\section{SySTEM DESCRIPTION AND DiRECT CONTROL ARCHITECTURE}

\section{A. MEMS Gyroscope Model}

The dynamic behavior of a MEMS gyroscope can be described through its governing differential equations, such as in [6], defining a multi-input multi-output (MIMO) linear system, denoted $G\left(\Omega_{z}\right)$, whose parameters depend on the 
angular rate $\Omega_{z}$. From the input-to-output perspective, we can consider the following representation:

$$
\left[\begin{array}{l}
x \\
y
\end{array}\right]=G\left(\Omega_{z}\right)\left[\begin{array}{l}
u_{x} \\
u_{y}
\end{array}\right]=\left[\begin{array}{ll}
G_{x x}\left(\Omega_{z}\right) & G_{x y}\left(\Omega_{z}\right) \\
G_{y x}\left(\Omega_{z}\right) & G_{y y}\left(\Omega_{z}\right)
\end{array}\right]\left[\begin{array}{l}
u_{x} \\
u_{y}
\end{array}\right],
$$

where the diagonal terms $G_{x x}$ and $G_{y y}$ correspond to the principal resonating modes (drive and sense) with resonance frequencies $\omega_{0_{x}}$ and $\omega_{0_{y}}$, and quality factors $Q_{x}$ and $Q_{y}$. The indices $x$ and $y$ denote the drive and sense modes, respectively. The off-diagonal terms $G_{x y}$ and $G_{y x}$ correspond to the coupling transfers between these two resonant modes. The input signal $u_{x}$ (respectively $u_{y}$ ) is a voltage proportional to the force applied to the drive (resp. sense) mode; and the output signal $x$ (resp. $y$ ) is a voltage proportional to the proof mass displacements along the $\vec{x}$ - and $\vec{y}$-axes.

\section{B. Closed-loop Operation}

In the closed-loop operation, the proof mass is driven to oscillate along the $\vec{x}$-axis following a sinusoidal reference signal $x_{r e f}$ of amplitude $A_{r e f}$ and frequency $\omega_{0}$, that is,

$$
x_{\text {ref }}(t)=A_{\text {ref }} \sin \left(\omega_{0} t\right) .
$$

Because of the high quality factor of the drive mode, $\omega_{0}$ is intended to be as close as possible to $\omega_{0_{x}}$ [6].

When the device is submitted to a nonzero angular rate $\Omega_{z}$, a Coriolis force appears, producing secondary oscillations along $\vec{y}$. This Coriolis force, denoted $F_{c y}$, is defined as

$$
F_{c y}(t) \propto \Omega_{z}(t) \cdot \dot{x}(t) .
$$

If $x$ tracks $x_{r e f}$, (2) can then be rewritten as

$$
F_{c y}(t) \propto \Omega_{z}(t) \cdot \cos \left(\omega_{0} t\right) .
$$

Therefore, it is possible to measure $\Omega_{z}$ by demodulating $F_{c y}$. In closed-loop, $F_{c y}$ is often estimated through the socalled force-to-rebalance strategy, which uses a controller to compensate for the Coriolis force, keeping $y(t) \equiv 0$. If the coupling between the drive and sense modes is negligible, the force $u_{y}$ required to keep $y(t) \equiv 0$ is then proportional to (3), and we can thus define the Coriolis force estimate $u_{e s t}=-u_{y} \propto F_{c y}$. If the cross-coupling cannot be neglected, ancillary control loops (quadrature compensation or others) might be necessary to provide an accurate measure.

To summarize, in order to obtain an accurate measure of $\Omega_{z}$, we need: (i) a good tracking of $x_{r e f}$ by $x$, and (ii) an accurate estimation of $F_{c y}$ by $u_{e s t}$.

\section{Direct Control Architecture}

To pursue the above requirements, we propose to use the direct control architecture, which is depicted in Fig. 1. It is composed of the MEMS gyroscope, a digital controller $K_{d}$ with digital-to-analog (DAC) and analog-to-digital converters (ADC) with sampling frequency $F_{s}$, and a demodulator. The to-be-controlled system (DACs, ADCs and $G$ ) is denoted $G_{d}$.

In the direct control architecture, the controller $K_{d}$, based on the signals $x_{r e f}, x$ and $y$, directly applies the control signals $u_{x}$ and $u_{y}$ to the drive and sense modes, respectively. We

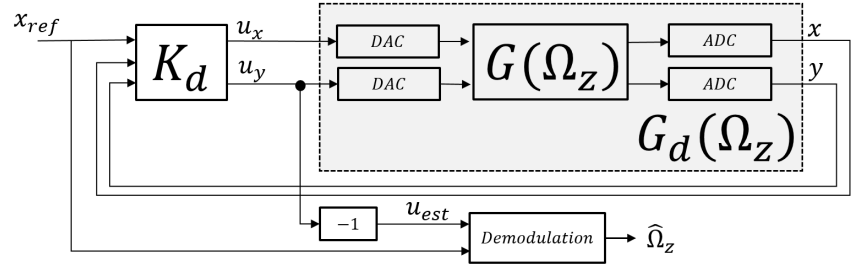

Fig. 1. Direct control architecture with a digital controller.

also highlight that the controller is a MIMO system. Hence, we have a single controller that operates the drive and sense modes simultaneously, without the need for ancillary feedback loops to deal with their couplings. This point is an essential advantage of this control architecture compared to those with individual control loops.

Another advantage of this architecture is that the demodulation is outside the loops, simplifying the electronic design. In addition, the to-be-controlled system $G_{d}$ is linear. This is not the case when considering the usual control architecture for the gyroscope operation. Taking the nonlinearities out of the closed-loop does not necessarily mean that the overall behavior of the sensor is improved in terms of nonlinearity. However, since the to-be-controlled system is linear, well-established control design methods can be employed, guaranteeing stability and performance for the closed-loop system.

This architecture may present some drawbacks as well. First, advanced control design methods may be required, and, depending on the method, the controller may present a high order, being more complex than PI-like controllers. Moreover, to respect the sampling theorem, while digital implementation, the sampling frequency $F_{s}$ must be at least the double of $\omega_{0 y}$ (here, $\omega_{0_{y}}>\omega_{0_{x}}$ ). This point can be a limitation for gyroscopes whose resonance frequencies are too high. Finally, the frequency of the reference signal does not keep track of $\omega_{0_{x}}$. An external mechanism would be needed to track variations of $\omega_{0_{x}}$. Despite these drawbacks, the above advantages make this control architecture an interesting alternative. Its design and implementation are the focus of the following sections.

\section{iII. Multivariable Digital Control Design}

\section{A. Control Design Towards Digital Implementation}

The presence of ADCs and DCAs modifies the dynamics of the to-be-controlled system $G_{d}$ with respect to $G$. Thus, they have to be properly considered for the controller design. Here, the gyroscope model $G_{d}$ is obtained through system identification [8], [9], taking into account the effects of the ADCs and DACs. The steps to design a digital controller are thus summarized:

1) Given a discrete-time (DT) model $G_{d}$, a continuoustime (CT) model $G_{c}$ is obtained through the bilinear (or Tustin) transform.

2) Based on $G_{c}$, a CT controller $K_{c}$ is designed. Here, we use the $H_{\infty}$ synthesis, detailed in the next subsection.

3) The DT model $K_{d}$ of the controller is finally obtained through the bilinear transform of $K_{c}$.

Further details can be found in [10] and references therein. 


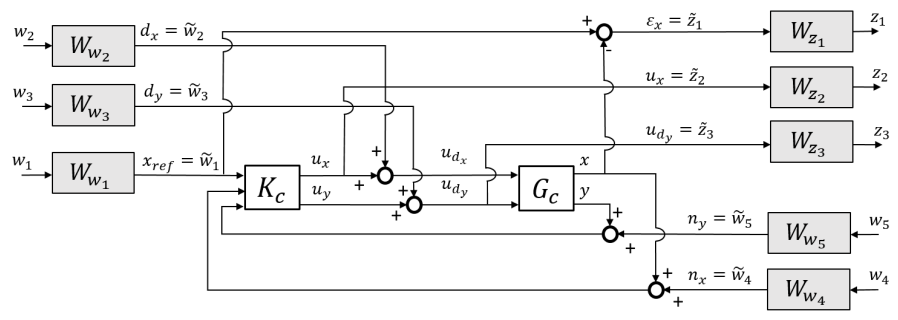

Fig. 2. $H_{\infty}$ criterion.

\section{B. $H_{\infty}$ Synthesis}

In this work, we adopt the $H_{\infty}$ synthesis, in which the controller design is formulated as an optimization problem subject to mathematical constraints. These constraints express stability, performance and robustness requirements as a mathematical criterion to be minimized. Here, we present the most relevant aspects of the method. For further details, we refer the interested reader to [11], for instance.

For the controller design, the Coriolis forces are assumed to be exogenous disturbances $d_{x}$ and $d_{y}$ of the type

$$
\begin{aligned}
& d_{x}(t)=A_{d_{x}} \sin \left(\omega_{0} t+\phi_{x}\right) \\
& d_{y}(t)=A_{d_{y}} \sin \left(\omega_{0} t+\phi_{y}\right),
\end{aligned}
$$

where $A_{d_{x}} \in \mathbb{R}, \phi_{x} \in \mathbb{R}, A_{d_{y}} \in \mathbb{R}$ and $\phi_{y} \in \mathbb{R}$. In this case, $G_{c}$ becomes a linear time-invariant (LTI) system (computed with $\Omega_{z}=0$ ). Then, the estimation specification can be recast as the rejection of the disturbance $d_{y}$ on the estimation error. Indeed, if we define $u_{e s t}=-u_{y}$ and the estimation error $u_{d_{y}}=d_{y}-u_{e s t}=d_{y}+u_{y}$, by rejecting $d_{y}$ on $u_{d_{y}}$, $u_{e s t}$ tends to the Coriolis force $d_{y}$.

Based on the above discussion and on Sec. II-B, the main control objectives are defined as follows:

(i) reference tracking: $x$ tracks the signal $x_{r e f}$ of (1), i.e., $\varepsilon_{x} \triangleq x_{r e f}-x \rightarrow 0$

(ii) disturbance rejection: $d_{y}$ of (4) is rejected on the signal $u_{d_{y}}$, i.e., $u_{d_{y}} \rightarrow 0$.

For our application, we consider the scheme of Fig. 2, where we include the measurement noises $n_{x}$ and $n_{y}$ and define the signals of interest $\widetilde{w}=\left(x_{r e f}, d_{x}, d_{y}, n_{x}, n_{y}\right)^{T}$ and $\widetilde{z}=\left(\varepsilon_{x}, u_{x}, u_{d_{y}}\right)^{T}$, weighting functions $W_{w}=$ $\operatorname{diag}\left(W_{w_{1}}, \ldots W_{w_{5}}\right)$ and $W_{z}=\operatorname{diag}\left(W_{z_{1}}, \ldots W_{z_{3}}\right)$ such that $w=\left(w_{1}, w_{2}, w_{3}, w_{4}, w_{5}\right)^{T}=W_{w}^{-1} \widetilde{w}$ and $z=$ $\left(z_{1}, z_{2}, z_{3}\right)^{T}=W_{z} \widetilde{z}$. The problem is then formulated as to compute a controller such that the closed-loop system of Fig. 2 is stable and

$$
\left\|T_{w \rightarrow z}(s)\right\|_{\infty}<\gamma,
$$

where $T_{a \rightarrow b}$ denotes the transfer from a signal $a$ to a signal $b$ and the variable $\gamma>0$ defines the performance level.

If this problem has a solution for $\gamma<1$, then (5) implies

$$
\begin{aligned}
& \forall \omega \in \mathbb{R}, \forall k \in\{1, \ldots, 5\}, \forall l \in\{1, \ldots, 3\}, \\
& \left|T_{\widetilde{w}_{k} \rightarrow \widetilde{z}_{l}}(j \omega)\right|<\left|W_{z_{l}}(j \omega) W_{w_{k}}(j \omega)\right|^{-1} .
\end{aligned}
$$

Hence, it is possible, via a judicious choice of the weighting functions, to impose upper bounds on $\left|T_{\widetilde{w}_{k} \rightarrow \widetilde{z}_{l}}(j \omega)\right|$, and, as a consequence, the desired control specifications. Several specifications can thus be addressed, including robust stability. The complete description is available in [12]. In the sequel, we focus on the two main control objectives.

(i) Reference tracking: From (6) $(k=l=1)$, we have

$$
\forall \omega \in \mathbb{R}, \quad\left|T_{x_{r e f} \rightarrow \varepsilon_{x}}(j \omega)\right|<\left|W_{z_{1}}(j \omega) W_{w_{1}}(j \omega)\right|^{-1} .
$$

For $x_{r e f}$ given in (1) with $\omega_{0}=\omega_{0_{x}}$, the objective is to obtain $\left|\varepsilon_{x}\right|<\varepsilon_{x_{\max }}\left|A_{\text {ref }}\right|$ in steady-state, which is equivalent to $\left|T_{x_{r e f} \rightarrow \varepsilon_{x}}\left(j \omega_{0_{x}}\right)\right|<\varepsilon_{x_{\max }}$, where $\varepsilon_{x_{\max }} \in \mathbb{R}^{+}$is the maximum allowed relative tracking error. The weighting functions $W_{w_{1}}$ and $W_{z_{1}}$ are then chosen to express this control specification. For instance, we can use

$$
W_{w_{1}}(s) W_{z_{1}}(s)=k \frac{1+\alpha_{1} s+\beta s^{2}}{1+\alpha_{2} s+\beta s^{2}},
$$

where the parameters $k>0, \alpha_{1}>\alpha_{2}>0$ and $\beta>0$ are tuned such as $\left|W_{w_{1}}\left(j \omega_{0_{x}}\right) W_{z_{1}}\left(j \omega_{0_{x}}\right)\right|^{-1} \leq \varepsilon_{x_{\max }}$.

(ii) Disturbance rejection (Coriolis force estimation):

Similarly to the previous specification and from (6) with $k=$ $l=3, W_{z_{3}}$ and $W_{w_{3}}$ are chosen such as,

$$
\left|T_{d_{y} \rightarrow u_{d_{y}}}\left(j \omega_{0_{x}}\right)\right|<\left|W_{z_{3}}\left(j \omega_{0_{x}}\right) W_{w_{3}}\left(j \omega_{0_{x}}\right)\right|^{-1}<\varepsilon_{u_{\max }},
$$

where $\varepsilon_{u_{\max }} \in \mathbb{R}^{+}$is the maximum allowed estimation error with respect to the disturbance $d_{y}$.

\section{IMPLEMENTATION RESULTS}

The proposed approach is implemented on the Asygn platform AS3125-SDK, which contains a high-performance front-end IC and a high-speed FPGA, where the controller is programmed. We use $F_{s} \approx 5.5 \omega_{0_{x}} /(2 \pi)$. The gyroscope is a prototype adapted to the experimental protocol. Tests at rest and on a rotating table are made at room temperature. The purpose of these tests is to evaluate the implementation of the control loops and their efficiency. In particular, the reference tracking and the disturbance rejection specifications are evaluated. We consider the following specifications:

- reference tracking with $\varepsilon_{x_{\max }}=0.5 \%$;

- disturbance rejection with $\varepsilon_{u_{\max }}=1 \%$.

A DT MIMO model of the gyroscope is obtained by identification and the electrical coupling is compensated, as presented in [8]. The key parameters of the drive and sense modes are $\omega_{0_{x}}=2 \pi \cdot 11586 \mathrm{rad} / \mathrm{s}, \omega_{0_{y}}=2 \pi \cdot 11677 \mathrm{rad} / \mathrm{s}$, $Q_{x}=80502$ and $Q_{y}=8099$.

Based on this model and following the approach of Sec. III, we design a DT MIMO controller with $\gamma=0.95$. Further details on the identification results and on the controller design are provided in [12].

To verify the reference tracking specification, we apply a reference signal of the form of (1) with $A_{r e f}=0.5 \mathrm{~V}$. The results at rest $\left(\Omega_{z}=0\right)$ are presented in Fig. 3 (left), where we observe that the signals $x$ and $x_{r e f}$ are almost superposed, indicating a good reference tracking. The ratio of the power spectral densities of $\varepsilon_{x}$ and $x_{r e f}$ gives an estimate of $\left|T_{x_{r e f} \rightarrow \varepsilon_{x}}\right|$ and allows to evaluate the reference tracking 

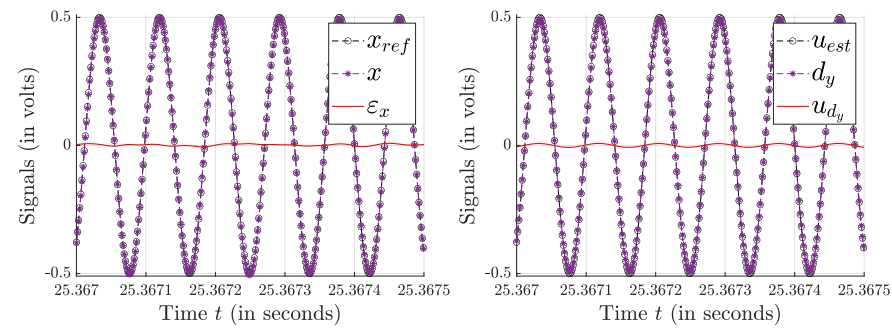

Fig. 3. Implementation results for the reference tracking (left) and for the disturbance rejection (right) at rest $\left(\Omega_{z}=0\right)$.
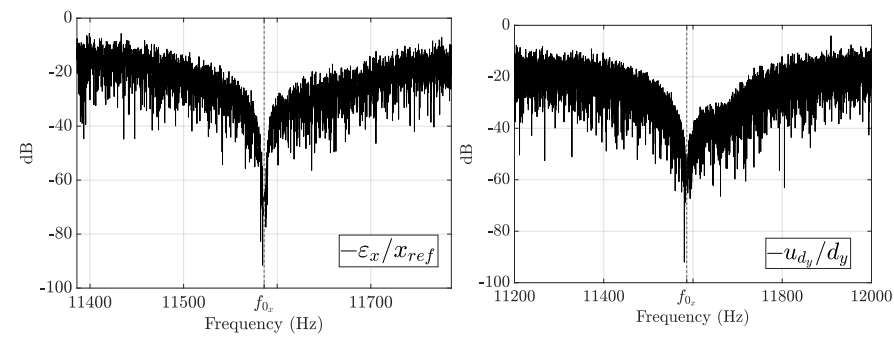

Fig. 4. Power spectral density estimates of the relative tracking error $\varepsilon_{x} / x_{r e f}$ (left) and of the relative estimation error $u_{d_{y}} / d_{y}$ (right) at rest $\left(\Omega_{z}=0\right)$.

specification. At the frequency $\omega_{0 x}$, Fig. 4 (left) reveals that this gain is around $-68 \mathrm{~dB}$, i.e., $0.04 \%<0.5 \%$, fulfilling the specification.

The estimation error cannot be directly obtained, since the real Coriolis force is unknown. To evaluate the estimation error, an artificial disturbance $d_{y}$ (see (4)) of amplitude $A_{d_{y}}=0.5 \mathrm{~V}$ is added on $u_{y}$. The results at rest $\left(\Omega_{z}=0\right)$ are presented in Fig. 3 (right) and an estimate of $\left|T_{d_{y} \rightarrow u_{d y}}\right|$ is presented in Fig. 4 (right), revealing a gain close to $-56 \mathrm{~dB}$, i.e., $0.16 \%<1 \%$. Therefore, the estimation specification is fulfilled as well.

We also test the closed-loop operation of the sensor and its performance on a rotating table with $\Omega_{z} \in$ $\{-300,-200,-100,100,200,300\}^{\circ} / \mathrm{s}$. Regarding the reference tracking and the disturbance rejection specifications for different angular rates, the results are similar to those with $\Omega_{z}=0$ (see Fig. 3 and Fig. 4), proving the validity of the proposed approach. Finally, the Coriolis force estimate $u_{e s t}$ is demodulated and multiplied by the scale factor, obtaining $\widehat{\Omega}_{z}$, which is compared to the actual $\Omega_{z}$ in Fig. 5. Although the estimation lacks of linearity (scale factor nonlinearity is around $3 \%$; the optimization of the MEMS prototype can probably improve it), these experiments validate the proposed concept.

\section{Conclusions And Perspectives}

In this work, we propose a design method for a multivariable digital controller of a MEMS gyroscope. The proposed method is based on the $H_{\infty}$ synthesis, which is a very flexible design method, allowing to express and to ensure different performance specifications in the frequency domain. These specifications are ensured through the choice of the weighting functions, which can be adapted by the designer.

Although the gyroscope prototype used for the test might be optimized to improve linearity of the output signal, the

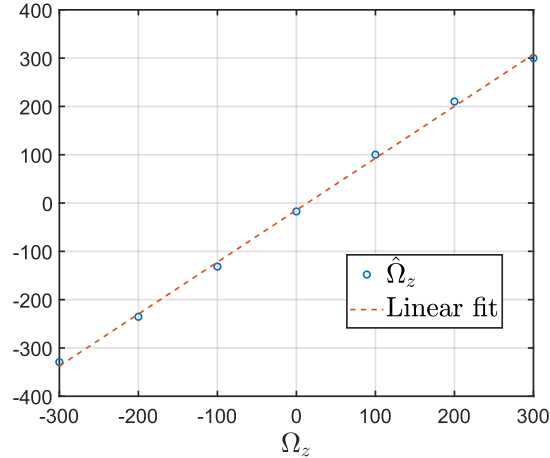

Fig. 5. Values of $\widehat{\Omega}_{z}$ compared to the true value of $\Omega_{z}$.

implementation results prove the implementability of the control architecture and constitute a first step towards a possible industrialization.

Some improvements are to be considered. Currently, we work on the tracking of $\omega_{0_{x}}$ in real time, such that the reference frequency and the controller can be parameterized by $\omega_{0}$. The first theoretical results on the controller parameterization can be found in [10].

Sometimes, relays are used to actuate the drive and sense modes, introducing a nonlinear element between the controller and the gyroscope, similarly to the EM- $\Sigma \Delta$ feedbacks. Our approach can also be considered in these cases, as in [5].

\section{REFERENCES}

[1] M. Egretzberger, F. Mair, and A. Kugi, "Model-based control concepts for vibratory MEMS gyroscopes," Mechatronics, vol. 22, no. 3, pp. 241250, Apr. 2012.

[2] Zurich Instruments, "Control of MEMS Coriolis vibratory gyroscopes," Zurich Instruments, Tech. Rep., 2015. [Online]. Available: https://www.zhinst.com/sites/default/files/zi_appnote_mems_gyroscope.pdf

[3] R. T. M'Closkey, A. Vakakis, and R. Gutierrez, "Mode localization induced by a nonlinear control loop," Nonlinear Dyn., vol. 25, no. 1/3, pp. 221-236, 2001.

[4] J. Raman, E. Cretu, P. Rombouts, and L. Weyten, "A closed-loop digitally controlled MEMS gyroscope with unconstrained sigma-delta force-feedback," IEEE Sens. J., vol. 9, no. 3, pp. 297-305, Mar. 2009.

[5] F. Saggin, A. Korniienko, G. Papin, E. Markiewicz, Y. David, A. El Hajj, and G. Scorletti, " $H_{\infty}$ Design of an EM- $\Sigma \Delta$ feedback for MEMS gyroscopes," in DGON Inertial Sensors and Systems (ISS). IEEE, 2020.

[6] A. M. Shkel, R. Horowitz, A. A. Seshia, S. Park, and R. T. Howe, "Dynamics and control of micromachined gyroscopes," in Proc. 1999 Am. Control Conf., vol. 3, no. June. IEEE, 1999, pp. 2119-2124.

[7] J. Fei and C. Batur, "Robust adaptive control for a MEMS vibratory gyroscope," Int. J. Adv. Manuf. Technol., vol. 42, pp. 293-300, 2009.

[8] K. Colin, F. Saggin, C. Le Blanc, X. Bombois, A. Korniienko, and G. Scorletti, "Identification-based approach for electrical coupling compensation in a MEMS gyroscope," in IEEE Int. Symp. Inert. Senso. (INERTIAL). IEEE, apr 2019.

[9] K. Colin, "Data informativity for the prediction error identification of MIMO systems: identification of a MEMS gyroscope," $\mathrm{PhD}$, Université de Lyon, Sep. 2020. [Online]. Available: https://tel.archivesouvertes.fr/tel-03114994

[10] F. Saggin, J. Ayala-Cuevas, A. Korniienko, and G. Scorletti, "Parameterdependent $H_{\infty}$ control for MEMS gyroscopes: synthesis and analysis," in 2020 IFAC World Congress, Berlin, Germany, Jul. 2020.

[11] S. Skogestad and I. Postlethwaite, Multivariable feedback control analisys and design, 2nd ed. John Wiley \& Sons, 2001.

[12] F. Saggin, C. Pernin, A. Korniienko, G. Scorletti, and C. Le Blanc, "On the digital control of MEMS gyroscopes: a robust approach," Ecole Centrale de Lyon, Research Report, Feb. 2021. [Online]. Available: https://hal.archives-ouvertes.fr/hal-03125932 